\title{
The Role of the Right Cerebral Hemisphere in Processing Novel Metaphoric Expressions: A Transcranial Magnetic Stimulation Study
}

\author{
Gorana Pobric ${ }^{1}$, Nira Mashal ${ }^{2}$, Miriam Faust ${ }^{2}$, and Michal Lavidor ${ }^{1}$
}

\begin{abstract}
Previous research suggests that the right hemisphere $(\mathrm{RH})$ may contribute uniquely to the processing of metaphoric language. However, causal relationships between local brain activity in the $\mathrm{RH}$ and metaphors comprehension were never established. In addition, most studies have focused on familiar metaphoric expressions which might be processed similarly to any conventional word combination. The present study was designed to overcome these two problems by employing repetitive transcranial magnetic stimulation (rTMS) to examine the role of the $\mathrm{RH}$ in processing novel metaphoric expressions taken from poetry. Right-handed participants were presented with four types of word pairs, literal, conventional metaphoric and novel metaphoric expressions, and unrelated word pairs, and were asked to perform a semantic judgment task. rTMS of the right posterior superior temporal sulcus disrupted pro-
\end{abstract}

\section{INTRODUCTION}

The accumulated evidence from neurologically intact, split brain, and brain-injured participants indicates that the left (LH) and right (RH) cerebral hemispheres represent parallel and distinct semantic processing systems and that each of these systems contributes uniquely to the interpretation of linguistic material (e.g., Floel, Buyx, Breitenstein, Lohmann, \& Knecht, 2005; Chiarello, 2003; Faust \& Lavidor, 2003; Stephan et al., 2003; Gazzaniga, 2000; Beeman, 1998). However, whereas a causal role for LH regions in semantic processing was demonstrated in recent transcranial magnetic stimulation (TMS) studies (Vallesi, Shallice, \& Walsh, 2007; Nixon, Lazarova, Hodinott-Hill, Gough, \& Passingham, 2004; Devlin, Matthews, \& Rushworth, 2003; Oliveri et al., 2001), complementary findings regarding RH processing were not studied (except Oliveri et al., 2001), although fMRI studies established an RH involvement in various semantic tasks (Mashal, Faust, Hendler, \& Jung-Beeman, 2007; Eviatar \& Just, 2006; Mashal, Faust, \& Hendler, 2005; Jung-Beeman et al., 2004). We therefore sought to examine the role of the $\mathrm{LH}$ and $\mathrm{RH}$ in semantic process-

${ }^{1}$ University of Hull, UK, ${ }^{2}$ Bar-Ilan University, Ramat-Gan, Israel cessing of novel but not conventional metaphors, whereas rTMS over the left inferior frontal gyrus selectively impaired processing of literal word pairs and conventional but not novel metaphors (Experiment 1). In a further experiment, we showed that these effects were due to right-left asymmetries rather than posterior-anterior differences (Experiment 2). This is the first demonstration of TMS-induced impairment in processing novel metaphoric expressions, and as such, confirms the specialization of the $\mathrm{RH}$ in the activation of a broader range of related meanings than the left hemisphere, including novel, nonsalient meanings. The findings thus suggest that the $\mathrm{RH}$ may be critically involved in at least one important component of novel metaphor comprehension, the integration of the individual meanings of two seemingly unrelated concepts into a meaningful metaphoric expression.

ing by assessing the efficiency of metaphorical expressions comprehension when the functioning of the LH and $\mathrm{RH}$ was suppressed by TMS of the inferior frontal gyrus (IFG) and the posterior superior temporal sulcus (STS).

Metaphor comprehension involves some kind of meaning extension through the establishment of correspondence between concepts from disparate knowledge domains, as opposed to literal comparisons involving two things that are clearly alike (Cacciary \& Glucksberg, 1994; for a review, see Bowdle \& Gentner, 2005). According to Beck (1987), the understanding of metaphors "forces the mind" to construct a high-order mental linkage of two different, frequently unrelated, category domains, the topic and the vehicle (e.g., "job" and "jail"). There is much evidence that the semantic system of the RH specializes in integrating distantly related semantic information (Jung-Beeman, 2005; Jung-Beeman et al., 2004; Chiarello, 2003; Faust \& Lavidor, 2003; Beeman, 1998; Brownell, Simpson, Bihrle, Potter, \& Gardner, 1990). Therefore, this view of $\mathrm{RH}$ language comprehension is consistent with a selective role for the RH in processing metaphoric language (see, e.g., Mashal et al., 2007; Burgess \& Chiarello, 1996, for reviews). Although this mode of multiple meaning activation seems to be important for the 
successful processing of conventional, relatively familiar, metaphors, it may be even more critical for the comprehension of novel, less familiar metaphors, such as metaphoric expressions taken from poetry (Mashal et al., 2005).

Most previous research linking metaphor processing to the RH has contrasted literal semantic relations with conventional, relatively familiar, metaphoric relations. Although some research using conventional metaphoric stimuli appears to support an enhanced role of the $\mathrm{RH}$ in processing metaphoric language (e.g., Sotillo et al., 2005; Rinaldi, Marangolo, \& Baldassari, 2004; Beeman, 1998; Bottini et al., 1994; Chiarello, 1991), other studies report either LH superiority or no hemispheric differences in metaphor comprehension (see, e.g., Ahrens et al., 2007; Oliveri, Romero, \& Papagno, 2004; Rapp, Leube, Erb, Grodd, \& Kircher, 2004; Giora, Zaidel, Soroker, Batori, \& Kasher, 2000; Chobor \& Schweiger, 1998; Tompkins, 1990).

An explanation of the $\mathrm{RH}$ in metaphor processing has been provided by the coarse semantic coding theory developed by Jung-Beeman (2005), Jung-Beeman et al. (2004), and Beeman (1998). According to the coarse coding theory, immediately after encountering a word, the LH engages in relatively fine semantic coding, strongly focusing on a few closely related word meanings or semantic features, whereas the $\mathrm{RH}$ engages in coarse semantic coding, weakly and diffusely activating large semantic fields containing multiple alternative meanings and more distant associates. The larger and less discriminate the semantic field, the more likely it is to overlap with semantic fields activated by other input words, including metaphorically related words. Because the metaphorical meaning of a word is usually more semantically distant than its literal meaning, the processing of a metaphoric expression may depend on the ability to activate a broader, more flexible set of semantic associations in order to integrate the meanings of the weakly related words into a meaningful linguistic expression. Thus, the coarse coding theory postulates that $\mathrm{RH}$ semantic processes are, in general, suited for the processing of any distant semantic relations be they literal or metaphorical. However, because the comprehension of novel metaphors requires the establishment of a mental linkage between two concepts ("conscience," "storm") that are distantly related, the integration of these concepts into a meaningful expression ("conscience storm") requires the RH coarse coding. According to this explanation, semantic features such as "turbulent," "unstable," "not calm," which are activated by the term "storm," are only distantly related to the term "conscience." However, the coarse semantic coding of the RH elicits a large and diffuse semantic field that may include these semantic features and, consequently, the semantic fields for "storm" and "conscience" are more likely to overlap. As a result of this meaning overlap, the $\mathrm{RH}$ is more sensitive than the LH to the distant semantic relations between "storm" and "conscience" and can integrate the meanings of the two concepts in order to form a meaningful novel metaphoric expression (i.e., turbulent conscience). In contrast, the fine semantic coding of the LH elicits a small and focused semantic field including only closely related associates of the term "conscience" (i.e., moral). The small semantic field in the LH are less likely to overlap and, consequently, the $\mathrm{LH}$ is less efficient in integrating the meanings of two distantly related concepts into a novel meaningful expression. We argue that the coarse coding of the $\mathrm{RH}$ is more compatible with the comprehension of novel metaphoric expressions than with the comprehension of both familiar metaphoric and literal expressions. Processing literal expressions (e.g., "snow storm”) may not depend on the coarse coding of the $\mathrm{RH}$ because the concepts ("snow," "storm") are closely related (both derived from the same semantic category, e.g., "winter"). In addition, processing familiar metaphoric expressions (e.g., "velvet voice") may also not require the $\mathrm{RH}$ coarse coding mechanism because the integration of the two concepts ("velvet," "voice") has already been established through the frequent use of the language.

Alternatively, the distinction between conventional and novel metaphoric expressions with regard to the differential processing by the $\mathrm{LH}$ and $\mathrm{RH}$, respectively, is consistent with the graded salience hypothesis developed by Giora (1997, 2002). According to this theory, it is the degree of semantic salience (i.e., familiarity, frequency, prominence, conventionality, prototypicality, contextual independence) rather than the literal/ metaphorical meaning of a linguistic expression that determines processing. The graded salience hypothesis suggests that meanings of linguistic expressions that are more salient, be they literal or figurative, are processed before linguistic expressions that are less salient, whereas linguistic expressions that are equally salient are processed simultaneously (as shown by Giora \& Fein, 1999a, 1999b). According to this model, the LH/literal meaning versus $\mathrm{RH} /$ metaphoric meaning dichotomy may be replaced by an $\mathrm{LH} /$ salient meanings versus $\mathrm{RH} /$ nonsalient meanings dichotomy.

The integration of the coarse coding model with the graded salience hypothesis could imply that although a few salient literal or metaphorical meanings may be quickly activated in the $\mathrm{LH}$, the $\mathrm{RH}$ activates and maintains multiple nonsalient, either literal or metaphorical, meanings. Indeed, Schmidt, DeBuse, and Seger (2007) found an RH advantage for both metaphorical and literal unfamiliar sentences containing distant associates and LH advantage for familiar sentences containing closely related words. Furthermore, a recent fMRI study (Mashal et al., 2005) compared the patterns of brain activation induced by processing the meanings of literal, conventional metaphoric, novel metaphoric, and unrelated word pairs. The results indicated that the right homologue of Wernicke's area has a special role in processing 
unfamiliar novel metaphors. However, it is still unclear whether the region that was selectively active during novel metaphors processing (Mashal et al., 2007) has a causal role in semantic processing. To resolve this, the current study employed the only research method which enables establishing a causal model in healthy brains, the TMS.

The basis for this study has its precedence in previous work which used TMS to suppress semantic processing. Performance on several different types of semantic processing tasks has been shown to be impaired with magnetic stimulation of various regions in the LH. For instance, stimulation of the anterior left IFG increased reaction times (RTs) relative to no stimulation when subjects focused on the meaning of a visually presented word (Devlin et al., 2003). In contrast, stimulation had no effect when subjects attended to visual properties of the word on the screen. Other TMS studies of various semantic tasks confirmed the role of Wernicke's area in sentences comprehension (Andoh et al., 2006), the role of the anterior left IFG in synonym judgments (Gough, Nobre, \& Devlin, 2005), the role of the left posterior BA 37 in picture naming (Stewart, Meyer, Frith, \& Rothwell, 2001), and the role of left temporal repetitive TMS (rTMS) in impaired idiom comprehension (Oliveri et al., 2004).

These TMS studies have helped establish the casual role of the left IFG in semantic processing, however, they did not resolve the debate regarding the role of the $\mathrm{RH}$ in processing novel semantic associations. This is due to the fact that all the semantic tasks in the TMS studies tested semantic knowledge of familiar words or objects. To the best of our knowledge, previous TMS studies never reported any semantic role of the RH. In contrast, the fMRI findings (Mashal et al., 2005, 2007), in accordance with the integration of the coarse coding model and the graded salience hypothesis, lead to some very precise predictions regarding the RH semantic processing that can be tested in a TMS study. In particular, based on results from region-of-interest (ROI) analysis in the left IFG (Mashal et al., 2007), greater percent signal change was found for conventional metaphors than unrelated expressions, and marginally greater percent signal change for conventional metaphors than literal expressions, and greater percent signal change for novel metaphoric expressions than unrelated word pairs. Based on these results, we expected that TMS over the left IFG will gradually impair conventional metaphoric expressions, literal expressions, and novel metaphoric expressions in descending order (i.e., greater effect on the conventional metaphors than the literal expressions and greater effect on the literal expressions than the novel metaphoric expressions).

We applied TMS over the right Wernicke's area (more precisely over the right posterior superior temporal sulcus [rPSTS]), the left IFG, and a control stimulation over the vertex during processing of word pairs that were literally, metaphorically (conventional or novel), or not associated. Based on the predictions of the graded salience hypothesis and on the fMRI findings on differential patterns of semantic processing by the two cerebral hemispheres (Mashal et al., 2005, 2007), the main hypothesis of the present study was that TMS over the rPSTS will impair a semantic judgment task for novel metaphoric, but not for conventional metaphoric, expressions. Complementary, TMS over the left IFG will impair conventional metaphoric expressions and literal expressions with possible greater effect on the conventional metaphoric expressions than the literal expressions.

\section{EXPERIMENT 1: RIGHT PSTS AND LEFT IFG}

\section{Methods}

Design

We used a $3 \times 4$ factorial design with TMS (rPSTS, left IFG, and vertex) and expression type (literal, conventional metaphor, novel metaphor, and unrelated) as the two within-subjects factors. rTMS was administered in alternating blocks of single hemisphere stimulation. All other variables were randomly applied. Vertex rTMS trials were included to assess the potential effects that the auditory and tactile stimulation from the coil might have on performance. Vertex stimulation does not produce measurable evoked potentials or rCBF changes and was never related to semantic processing (Loo et al., 2000).

\section{Participants}

Twelve native English speakers took part in the experiment (mean age 24.1, $S D=6.2 ; 6$ men). All participants were right-handed, yielding a laterality quotient of at least +90 on the Edinburgh Handedness Inventory (Oldfield, 1971). All participants reported normal or corrected-to-normal vision and were naive to the purposes of the experiment. Before taking part in the experiment, participants were provided an information leaflet that explained the procedure to be used and were given at least 24 hours to decide whether they wished to participate. All participants signed a consent form and reported absence of epilepsy, or any other neurological conditions in themselves and their family. Local ethical committee approval was granted for all procedures. An additional 12 participants (mean age 25, $S D=5.3 ; 5$ men) were tested in a no-TMS condition for a further control condition.

\section{Stimuli and Apparatus}

Stimuli included 192 pairs of words that formed four types of semantic relations: literal (LP), such as breaking 
glass; conventional metaphoric (CM), such as defense line; novel metaphoric (NM), such as burning lie; or unrelated (UP), such as bouncing coal. Stimuli were balanced between conditions according to word length (mean 5.95 letters), frequency (mean 88 per million based on the validated Kucera-Frances norms at the English Lexicon Project; Balota et al., 2002), concreteness (mean $=415$, based on the MRC database; Coltheart, 1981), and part of speech. The mean length, frequency, and concreteness values of the two words in a pair were matched between the four different stimuli types such that no significant differences were found between conditions for the length, frequency, and the concreteness rate [all $F(3,45)<2$ ]. Prior to the study, we generated 240 pairs of words, 60 pairs for each type of semantic association, where the novel metaphors were taken from English modern poetry, and some were translated from Hebrew poetry metaphors (Mashal et al., 2005). The initial list of 240 pairs was submitted to 10 judges who did not participate in the study. These judges were asked to decide whether each expression is literally plausible, metaphorically plausible, or unrelated. Expressions that were rated by at least $75 \%$ of the judges as metaphorically/literally plausible or unrelated were selected for use in the corresponding conditions.

Another group of six judges received a list of only the metaphors rated as plausible in the first pretest, and were asked to rate their degree of familiarity on a 5-point familiarity scale ranging from 1 (bighly unfamiliar) to 5 (bighly familiar). Metaphoric expressions scoring less than 3 on the familiarity scale were selected for the study as novel metaphors (rating average 1.79), whereas those scoring above 3 on this scale were selected as conventional metaphors (rating average 4.32). In this way, we could distinguish between novel and conventional metaphors.

Subsequent to performing the filtering procedures described above, we compiled a final list of 192 word pairs, 48 pairs for each category (literal, conventional metaphors, novel metaphors, and unrelated pairs). The 192 word pairs were divided to three equal-size sets of 64 word pairs. Each subset contained 16 word pairs from each of the four semantic categories. The three subsets were rotated across the three TMS conditions in a Latinsquare design, such that each participant saw each word pair only once.

Because linguistically naive persons may have considerable difficulties making an explicit distinction between literal and metaphoric expressions, participants were trained to perform the same task on a different set of stimuli prior to the experiment. We explained to the participants that the meanings of metaphorical expressions contain an interpretation, which lies beyond the individual meanings of the words. For example, the expression "sweet sleep" is metaphorical because sleep is not really sweet (as opposed to "sweet cake"). We instructed the participants to pay attention to conven- tional metaphors, which seem literally related because of frequent daily use. We also informed them that some of the expressions are taken from poetry and that they might seem unfamiliar and meaningless ("burning lie") but still might have metaphorical meanings. The experimenter provided participants with many examples, until the participants understood clearly the distinction between literally and metaphorically related expressions.

\section{TMS Equipment and Protocol}

The target locations for rTMS were taken from the relevant fMRI study (Mashal et al., 2007). Sites for TMS were determined by collecting T1 magnetic resonance (MR) images on each subject. MR images were acquired on a 3-Tesla Siemens scanner prior to the TMS session. The images were reconstructed into a surface rendering of sulcal anatomy (BrainSight; www.magstim.co.uk) and the sites of stimulation were guided by the use of frameless stereotaxy system (Polaris). The target locations for rTMS were the rPSTS $(49,-28,4)$, the left IFG $(-42,12$, $5)$, and the vertex. We determined the location of the vertex by finding the intersection of the midsagittal plane (defined by nasion to inion line) and the midcoronal plane (defined by the line between the intertrachial notches of the ears). rTMS was performed using a Magstim Super-Rapid transcranial stimulator (Magstim, Whitland, UK) with a 40-mm figure-of-eight coil. The coil was placed such that the maximal induced current flowed in the anterolateral direction for the IFG, and downward for the rPSTS.

Motor threshold (MT) was defined as a minimal intensity of stimulation capable of inducing motor-evoked potentials following unilateral TMS over the motor cortex in the contralateral first dorsal interosseous (FDI) muscle greater than $50 \mu \mathrm{V}$ peak-to-peak amplitude in at least 6 out of 10 trials. The coil was held to the skull by the experimenter using one hand to hold the coil, and the other hand to stabilize the head against the coil. Repetitive pulse TMS (rTMS) was delivered at $10 \mathrm{~Hz}$ for $500 \mathrm{msec}$, starting at $200 \mathrm{msec}$ after the stimulus presentation. The average MT was $58 \%$ of the maximal stimulator output and the average stimulation intensity during rTMS was 64\% (the output strength of the TMS was set at $110 \%$ of the subject's MT).

\section{Procedure}

The experimental sessions consisted of three blocks of 64 trials each, one block for each TMS condition (left IFG, rPSTS, and vertex). Order of blocks was counterbalanced across subjects. The participants performed a semantic judgment task in front of a computer screen during the TMS stimulation. During each block, subjects viewed a black fixation point on a light gray background for $500 \mathrm{msec}$, which was replaced by a pair of words that were presented in screen center for $2000 \mathrm{msec}$. The 
stimuli were presented in a black 16-point Arial font. These pairs of words included familiar metaphoric expressions (conventional metaphors), novel metaphoric expressions, literal expressions, and unrelated word pairs, and stimuli type was random. Each stimulus was presented for 2000 msec followed by 3000 msec blank, to allow sufficient interval between stimulation trains. The train of magnetic pulses was given 200 msec following stimuli presentation, and lasted $500 \mathrm{msec}$. Participants were asked to indicate (by pressing one of three mouse buttons) whether the two words presented simultaneously at the center of a computer screen were related literally, metaphorically, or unrelated. The subjects were informed that some of the word pairs represent novel metaphoric expressions taken from poetry, and conducted a 32-trial training session (with word pairs that did not pass judgments) to ensure they could do the task reliably. Each experimental condition (a combination of TMS condition and type of word pair) repeated 16 times.

\section{Results}

Seven word pairs out of the 192 pairs (from all categories) that were responded to with less than 33\% correct responses (chance level) were not included in the analysis. Two repeated-measure analyses of variance (ANOVAs) with TMS condition (rPSTS, left IFG, and vertex) and semantic association type (literal, conventional metaphor, novel metaphor, and unrelated) as the withinsubjects factors were conducted with mean RTs and error rates as the dependent variables. For the F2 analysis, two mixed-design ANOVAs were conducted, with TMS condition as the within-subjects factor, semantic association type as the between-subject factor, and mean RTs and error rates as the dependent variables. Mean RTs and error rates are summarized in Table 1 . In addition, Table 1 summarizes the performance of the additional 12 subjects that were tested only in a no-TMS condition.

\section{Reaction Times}

RT analysis yielded a main effect of semantic relationship $[F 1(3,33)=10.19, p<.001 ; F 2(3,156)=3.01, p<$ $.05]$. Post hoc Bonferroni comparisons $(p<.05)$ revealed that RTs to novel metaphors (mean $=911 \mathrm{msec}$ ) were significantly slower than RTs to the other word pairs. RTs to conventional metaphors (mean $=755 \mathrm{msec}$ ) and to literally related word pairs (mean $=789 \mathrm{msec}$ ) did not significantly differ, and they were both faster than RT to unrelated word pairs (mean $=849 \mathrm{msec}$ ).

Crucially, there was a significant interaction between TMS condition and semantic relationship $[F 1(6,66)=$ $2.88, p<.05 ; F 2(6,312)=3.78, p<.01]$. Simple main effect analysis revealed that significant interference TMS effects were created for literal and conventional meta-
Table 1. Mean Reaction Times (and Standard Deviations) to Word Pairs (msec) and Error Scores as a Function of Type of Semantic Association and Site of rTMS

\begin{tabular}{lcccc}
\hline & Literal & $\begin{array}{c}\text { Conventional } \\
\text { Metaphor }\end{array}$ & $\begin{array}{c}\text { Novel } \\
\text { Metaphor }\end{array}$ & Unrelated \\
\hline \multicolumn{2}{l}{ TMS_Right PSTS } & & & \\
Mean RT & 798 & 744 & 988 & 870 \\
SD & 50 & 45 & 74 & 49 \\
$\%$ error & 16.4 & 30.6 & 39.9 & 17.1
\end{tabular}

TMS-Left IFG

$\begin{array}{lcccc}\text { Mean RT } & 811 & 781 & 870 & 832 \\ S D & 52 & 46 & 57 & 51 \\ \text { \% error } & 20.9 & 31.7 & 38.4 & 12.9\end{array}$

Control TMS (Vertex)

$\begin{array}{lcrrr}\text { Mean RT } & 759 & 741 & 877 & 845 \\ S D & 52 & 45 & 52 & 56 \\ \text { \% error } & 26.3 & 19 & 44 & 18\end{array}$

No TMS (Fresh 12 Subjects)

\begin{tabular}{lrrrr} 
Mean RT & 744 & 742 & 859 & 828 \\
SD & 77 & 80 & 78 & 98 \\
\% error & 15.5 & 12 & 14 & 16 \\
\hline
\end{tabular}

The semantic associations between pairs of words included familiar metaphoric expressions (conventional metaphors), novel metaphoric expressions, literal expressions, and unrelated word pairs (Experiment 1).

phorically related word pairs when TMS was applied over the left IFG in comparison to the control condition TMS over the vertex. TMS over the rPSTS caused a nonsignificant inhibition for literally related word pairs, and a significant inhibition $(110 \mathrm{msec}, p<.01)$ for novel metaphors (see Table 1 and Figure 1). A mixed-design analysis that compared the four semantic association types between the vertex condition and the no-TMS condition of the additional subjects revealed neither significant effects nor interactions.

\section{Percent of Correct Response (Accuracy)}

Accuracy analysis yielded a main effect of semantic relationship $[F 1(3,33)=12.1, p<.001 ; F 2(3,156)=2.99$, $p<.05]$. Post hoc Bonferroni comparisons $(p<.05)$ revealed that percent of correct responses to novel metaphors $($ mean $=59.4 \%$ ) was significantly lower than to the other word pairs (chance level was 33.3\%). Accuracy to conventional metaphors (mean $=73.3 \%$ ) and to literally related word pairs $($ mean $=78.9 \%)$ did not 


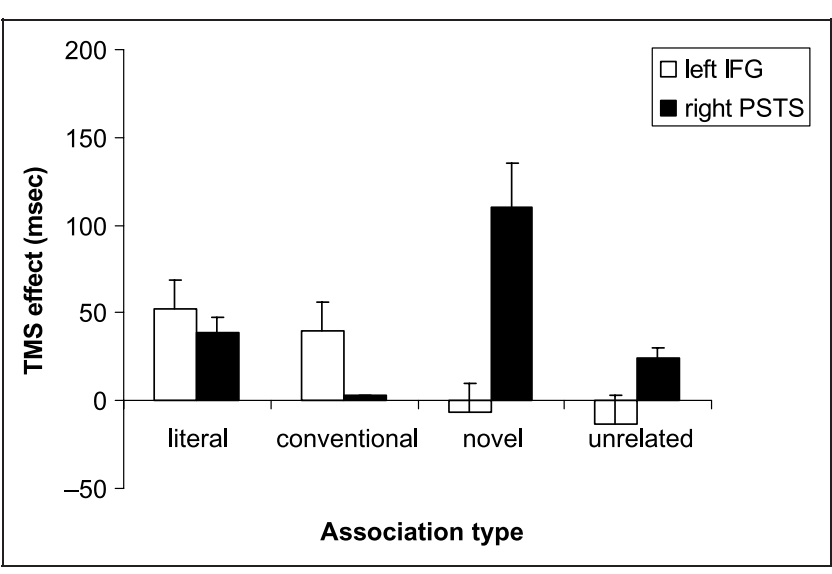

Figure 1. rTMS effect size as a function of semantic association type. TMS effect is the RT change (msec) caused by TMS. For example, the black bar above the "novel metaphor" category indicates that TMS in the RH caused an RT delay of $110 \mathrm{msec}$ for novel metaphors targets, when compared to the vertex TMS condition. Negative figures indicate RTs on TMS trials were faster than control trials (vertex). The semantic associations between pairs of words included familiar metaphoric expressions (conventional metaphors), novel metaphoric expressions, literal expressions, and unrelated word pairs (Experiment 1).

significantly differ, and was lower than accuracy to unrelated word pairs $($ mean $=84.2 \%$ ) $($ see Table 1$)$. There were no further significant effects or interactions.

When examining the distribution of errors (Table 2), only errors for literally associated pairs differed significantly as a function of the TMS condition $\left(\chi^{2}=23.1, d f=\right.$ $4, p<.05)$. Under the vertex TMS conditions, literal word pairs were mostly mistaken as metaphorically related, whereas TMS over the right or left site errors for literal pairs tended to include "unrelated" responses as well. Apart from this, the patterns under the different TMS conditions were similar, and showed that most errors for novel metaphors were "unrelated" responses, whereas most errors for conventional metaphors tended to be "literal" responses. Unrelated word pairs were typically mistaken for metaphors.

\section{Discussion}

Experiment 1 employed rTMS to examine the role of the $\mathrm{RH}$ and $\mathrm{LH}$ in processing conventional and novel metaphoric expressions taken from poetry. rTMS of the rPSTS disrupted processing of novel but not conventional metaphors, whereas rTMS over the left IFG selectively impaired processing of literal word pairs and conventional but not novel metaphors. This is the first demonstration of TMS-induced impairment in processing novel metaphoric expressions, and as such, supports the specialization of the $\mathrm{RH}$ in the activation of a broader range of related meanings than the $\mathrm{LH}$, including novel, nonsalient meanings.

Experiment 1 was designed in accordance with the significant ROI that were involved in processing novel and conventional metaphoric expressions in an fMRI study (Mashal et al., 2007). However, the left and right stimulation sites were not symmetrical in terms of posterior and anterior planes. Hence, there is a concern that the significant differences we report reflect, in fact, posterior/anterior asymmetries in responding to different types of semantic associations rather than left and right asymmetries. Although such a hypothesis is not supported by the fMRI results we reported (Mashal et al., 2007), it is, nevertheless, a threat to the interpretation we propose. We therefore added a further experiment (Experiment 2) that included the homologues right and left areas as well.

In Experiment 2, we applied TMS over the Wernicke's area and its right homologue (more precisely over the rPSTS), the left IFG and its right homologue, and a control stimulation over the vertex during processing of word pairs that were literally, metaphorically (conventional or novel), or not related. The main hypothesis of Experiment 2 was that TMS over the rPSTS will impair a semantic judgment task for novel metaphoric, but not for conventional metaphoric, expressions. Our fMRI data (Mashal et al., 2007) indicated that there was no main effect in the left PSTS to any of the linguistic stimuli. Both the PCA (networks) and the ROI analysis (Mashal et al., 2005, 2007; Eviatar \& Just, 2006) demonstrated that the left PSTS was activated for novel metaphors, conventional

Table 2. Error Distribution for All the Errors as a Function of Type of Semantic Association and Site of rTMS (Experiment 1)

\begin{tabular}{|c|c|c|c|c|}
\hline & Literal & $\begin{array}{l}\text { Conventional } \\
\text { Metaphor }\end{array}$ & $\begin{array}{c}\text { Novel } \\
\text { Metaphor }\end{array}$ & Unrelated \\
\hline \multicolumn{5}{|c|}{ TMS_Right PSTS Responses } \\
\hline No response & $9 \%$ & $11 \%$ & $10 \%$ & $10 \%$ \\
\hline "Metaphor" & $55 \%$ & 0 & 0 & $67 \%$ \\
\hline "Unrelated", & $35 \%$ & $9 \%$ & $75 \%$ & 0 \\
\hline "Literal" & 0 & $80 \%$ & $14 \%$ & $22 \%$ \\
\hline \multicolumn{5}{|l|}{ TMS - Left IFG } \\
\hline No response & $11 \%$ & $2 \%$ & $5 \%$ & $5 \%$ \\
\hline "Metaphor" & $49 \%$ & 0 & 0 & $65 \%$ \\
\hline "Unrelated" & $40 \%$ & $14 \%$ & $81 \%$ & 0 \\
\hline "Literal" & 0 & $84 \%$ & $14 \%$ & $30 \%$ \\
\hline \multicolumn{5}{|c|}{ Control TMS (Vertex) } \\
\hline No response & 0 & $6 \%$ & $5 \%$ & $12 \%$ \\
\hline "Metaphor" & $95 \%$ & 0 & 0 & $77 \%$ \\
\hline "Unrelated" & $5 \%$ & $7 \%$ & $81 \%$ & 0 \\
\hline "Literal" & 0 & $87 \%$ & $14 \%$ & $11 \%$ \\
\hline
\end{tabular}


metaphors, unrelated combinations, or literal expressions to the same extent. Complementary, TMS over the left IFG will impair conventional metaphoric expressions and literal expressions with possible greater effect on the conventional metaphoric expressions than the literal, expressions. We expected that TMS over the right IFG will mainly impair the processing of the conventional and the novel metaphoric expressions, but to a lesser extent as compared with the left IFG stimulation.

\section{EXPERIMENT 2: RIGHT AND LEFT PSTS, RIGHT AND LEFT IFG}

\section{Methods}

Design

We used a $5 \times 4$ factorial design, with TMS (rPSTS, left PSTS, right IFG, left IFG, and vertex) and semantic association type (literal, conventional metaphor, novel metaphor, and unrelated) as the two within-subjects factors. rTMS was administered in alternating blocks of single hemisphere stimulation. We had two vertex blocks in order to add control trials and to control number of stimuli repetitions. All other variables were randomly applied.

\section{Participants}

Ten native English speakers took part in the experiment (mean age $27.2, S D=3.0 ; 5$ men). All participants were right-handed, yielding a laterality quotient of at least +90 on the Edinburgh Handedness Inventory (Oldfield, 1971). All participants reported normal or corrected-tonormal vision and were naive to the purposes of the experiment. Before taking part in the experiment, participants were provided an information leaflet that explained the procedure to be used and were given at least 24 hours to decide whether they wished to participate. All participants signed a consent form and reported absence of epilepsy, or any other neurological conditions in themselves and their family. Local ethical committee approval was granted for all procedures.

\section{Stimuli and Apparatus}

Stimuli and apparatus were identical to the methods of Experiment 1, except that we repeated each stimulus twice along the six blocks of Experiment 2. Stimuli order was counterbalanced between the subjects.

\section{TMS Equipment and Protocol}

TMS protocols in Experiment 2 were identical to the protocols of Experiment 1. The target locations for rTMS were the rPSTS $(49,-28,4)$, left PSTS $(-46,-32,5)$, left IFG $(-42,12,5)$, right IFG $(42,21,5)$, and the vertex.

\section{Procedure}

The procedure in Experiment 2 was identical to Experiment 1's procedure apart from the additional three control conditions (right IFG, left PSTS, and an additional vertex condition), which resulted in a 3-hr experiment, including 25-min rest periods between the six sessions of 64 trials each.

\section{Results}

Eleven word pairs out of the 192 pairs (from all categories) that were responded to with less than 33\% correct responses (chance level) were not included in the analysis. Two repeated-measures ANOVAs with TMS condition (rPSTS, left PSTS, right IFG, left IFG, and vertex) and type of expression (literal, conventional metaphor, novel metaphor and unrelated) as the within-subjects factors were conducted with mean RTs and error rates as the dependent variables. For the F2 analysis, two mixed-design ANOVAs were conducted, with TMS condition as the within-subjects factor, expression type as the between-subject factor, and mean RTs and error rates as the dependent variables. Mean RTs and error rates are summarized in Table 3.

\section{Reaction Times}

RT analysis yielded a main effect of expression type $[F 1(3,27)=35.62, p<.0001 ; F 2(3,147)=4.83, p<$ $.01]$. Post hoc Bonferroni comparisons $(p<.05)$ revealed that RT to novel metaphors (mean $=992 \mathrm{msec}$ ) were significantly slower than RTs to the other word pairs. RT to conventional metaphors (mean $=812 \mathrm{msec})$ and to literally related word pairs (mean $=838 \mathrm{msec}$ ) did not significantly differ, and they were both faster than RT to unrelated word pairs (mean $=917 \mathrm{msec}$ ).

Crucially, there was a significant interaction between TMS condition and expression type $[F 1(12,108)=2.59$, $p<.05 ; F 2(12,294)=4.22, p<.001]$. In order to understand the sources of the interaction, we calculated the TMS effect size by subtracting mean RT in the control condition (vertex) from the ROI TMS condition (see Figure 2). Repeated-measure analysis for the RTdifference measure revealed a significant interaction $[F 1(9,81)=3.19, p<.05 ; F 2(9,441)=2.78, p<.05]$. Simple main effect analysis revealed that TMS over the rPSTS caused a significant inhibition (135 msec, $p<.01$ ) for novel metaphors, whereas TMS over the left PSTS caused facilitation in responses to novel metaphors (43 msec, $p<.05$ ). Significant interference TMS effects were created for conventional metaphoric and literal expressions when TMS was applied over the left and right IFG in comparison to the control condition (see Table 3 and Figure 2). 
Table 3. Mean Reaction Times (and Standard Deviations) to Word Pairs (msec) and Error Scores as a Function of Type of Semantic Association and Site of rTMS

\begin{tabular}{lcccc}
\hline & Literal & $\begin{array}{c}\text { Conventional } \\
\text { Metaphor }\end{array}$ & $\begin{array}{c}\text { Novel } \\
\text { Metaphor }\end{array}$ & Unrelated \\
\hline TMS_Right PSTS & & & \\
Mean RT & 847 & 799 & 1101 & 965 \\
SD & 71 & 62 & 70 & 55 \\
\% error & 13.3 & 26.7 & 41.2 & 16.7
\end{tabular}

TMS_Left PSTS

$\begin{array}{lcccc}\text { Mean RT } & 799 & 807 & 923 & 893 \\ \text { SD } & 51 & 58 & 71 & 73 \\ \text { \% error } & 15.3 & 27.5 & 38.3 & 17.2\end{array}$

TMS_Right IFG

$\begin{array}{lcccc}\text { Mean RT } & 873 & 829 & 975 & 895 \\ \text { SD } & 64 & 49 & 68 & 77 \\ \text { \% error } & 16.6 & 28.5 & 37.3 & 14.1\end{array}$

TMS - Left IFG

$\begin{array}{lcccc}\text { Mean RT } & 864 & 860 & 998 & 902 \\ S D & 80 & 55 & 59 & 67 \\ \text { \% error } & 15.8 & 21.4 & 31.4 & 13.3\end{array}$

Control TMS (Vertex)

\begin{tabular}{lcccc} 
Mean RT & 808 & 767 & 966 & 932 \\
$S D$ & 60 & 63 & 69 & 65 \\
\% error & 22.5 & 18.7 & 41.4 & 16.7 \\
\hline
\end{tabular}

The semantic associations between pairs of words included familiar metaphoric expressions (conventional metaphors), novel metaphoric expressions, literal expressions, and unrelated word pairs (Experiment 2).

\section{Percent of Correct Response (Accuracy)}

Accuracy analysis yielded a main effect of a expression type $[F 1(3,27)=14.2, p<.001 ; F 2(3,147)=3.75, p<$ $.05]$. Post hoc Bonferroni comparisons $(p<.05)$ revealed that percent of correct responses to novel metaphors (mean $=62.1 \%$ ) were significantly lower than that for the other word pairs (chance level was 33.3\%). Accuracy to conventional metaphors (mean $=75.4 \%$ ), to literal expressions $($ mean $=83.3 \%)$, and to unrelated pairs $($ mean $=84.4 \%$ ) did not significantly differ (see Table 3). There were no further significant effects or interactions.

\section{Discussion}

The results of Experiment 2 replicated the significant results of Experiment 1. In both experiments, rTMS of the rPSTS disrupted processing of novel but not conventional metaphors, whereas rTMS over the left and right IFG selectively impaired processing of literal word pairs and conventional but not novel metaphors. In addition, as expected, TMS over the left PSTS did not impair the semantic processing of the novel metaphoric expressions. RH involvement in processing novel metaphors demonstrated in both experiments cannot be attributed therefore to some posterior/anterior asymmetry in processing novel metaphors.

\section{GENERAL DISCUSSION}

The present study employed rTMS to examine the role of the RH and LH in processing conventional metaphors and novel metaphoric expressions taken from poetry. rTMS of the rPSTS disrupted processing of novel, but not conventional, metaphors, whereas rTMS over the left and right IFG selectively impaired processing of literal word pairs and conventional but not novel metaphors. In addition, as expected, TMS over the left PSTS did not impair the semantic processing of the novel metaphoric expressions. This is the first demonstration of RH TMS-induced impairment in semantic processing. Moreover, because this impairment was uncovered only for novel metaphoric expressions, the findings support the specialization of the $\mathrm{RH}$ in the activation of a broader range of related meanings than the $\mathrm{LH}$, including novel, nonsalient meanings.

We would like to draw attention to two main novel findings. One is the predicted interference due to rPSTS TMS stimulation that slowed down the processing of novel metaphoric expressions. The rPSTS has been in-

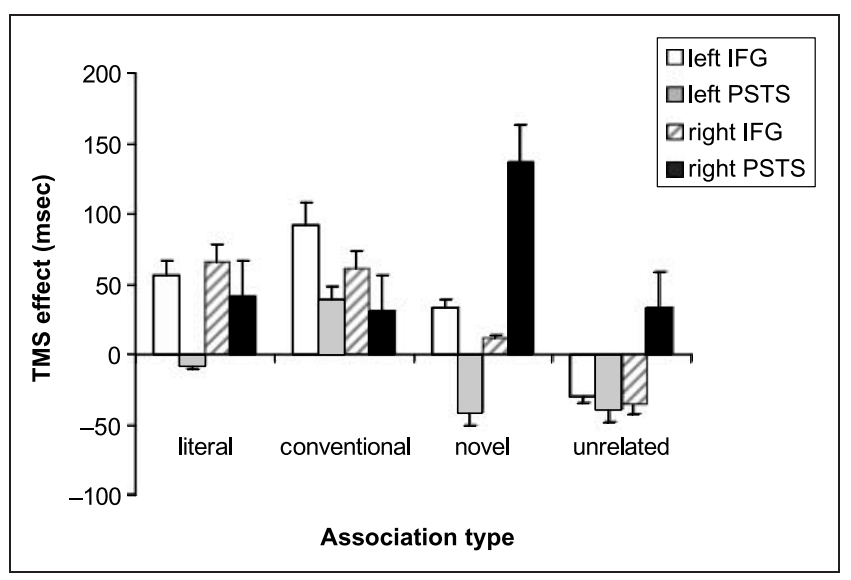

Figure 2. rTMS effect size as a function of semantic association type. TMS effect is the RT change (msec) caused by TMS. The semantic associations between pairs of words included familiar metaphoric expressions (conventional metaphors), novel metaphoric expressions, literal expressions, and unrelated word pairs (Experiment 2). 
volved when subjects processed novel metaphoric sentences as compared with literal sentences (Bottini et al., 1994), novel metaphoric expressions as compared with literal expressions (Mashal et al., 2005, 2007), novel ironic statements compared with literal sentences (Eviatar \& Just, 2006), as well as when subjects solved creative verbal problems (Jung-Beeman et al., 2004). In line with the coarse semantic coding model, it seems that the rPSTS is involved in processes that require the activation of large semantic fields, and thus, enable the integration of distantly related meanings (Schmidt et al., 2007). The unique involvement of the rPSTS in processing novel metaphoric expressions is also in line with the graded salience hypothesis (Giora, 2003) that predicts the involvement of the $\mathrm{RH}$ in processing nonsalient, unfamiliar, linguistic stimuli. It should be noted, however, that because both the unrelated word pairs and the novel metaphors are nonsalient to the same extent (both meanings are unfamiliar and thus not coded in the mental lexicon), the findings of the present study indicate that this involvement is restricted only to meaningful nonsalient meanings.

The other, unexpected, main finding of the present study is the facilitation in processing of novel metaphors following TMS over the left PSTS. This opposite pattern of facilitation with LH TMS may further support the claim regarding a right, and not left, hemisphere role for novel metaphors comprehension. In fact, previous studies have shown that TMS of one region may disinhibit the homologous regions in the contralateral hemisphere (Seyal, Ro, \& Rafal, 1995). This finding suggests that the stimulation of the left PSTS released an inhibitory effect of this region on the homologous rPSTS, resulting in faster comprehension of novel metaphoric expressions. This hypothesis is supported by Oliveri et al. (2004), who reported that stimulation over the right temporal lobe improved performance via disinhibition of the LH in a semantic task. According to this explanation, LH semantic processing mechanisms may interfere with the ability of the RH to integrate the individual meanings of two seemingly unrelated concepts into a meaningful metaphoric expression.

Through the use of rTMS, we can conclude that the $\mathrm{RH}$ plays a crucial role in processing novel metaphoric two word expressions. As noted in the Introduction, previous research on the processing of metaphoric language by the two cerebral hemispheres has yielded mixed results, and some studies failed to find $\mathrm{RH}$ involvement in the semantic processing of metaphors (e.g., Oliveri et al., 2004; Rapp et al., 2004). Our findings suggest that the absence of RH involvement in previous studies may be due to the nature of the particular metaphors applied. Thus, in the present study, the role of the RH was shown to be limited to novel metaphoric combinations. In contrast, the processing of familiar two-word combinations (whether metaphoric or literal pairs) was selectively impaired with rTMS over the left and right IFG. Another possible source for the inconsistent previous results may be the use of different types of linguistic stimuli. Whereas the present study used twoword metaphorical expressions, the available data obtained from neuroimaging studies reflect the processing of metaphoric sentences. Most of these studies found LH superiority (see, e.g., Stringaris, Medford, Giampetro, Brammer, \& David, 2007; Oliveri et al., 2004; Rapp et al., 2004) or bilateral involvement in metaphor comprehension (see, e.g., Ahrens et al., 2007), although some of the studies have shown RH superiority for processing metaphoric (e.g., Sotillo et al., 2005; Rinaldi et al., 2004; Bottini et al., 1994) and novel ironic sentences (Eviatar \& Just, 2006). However, as opposed to the present study that used two-word expressions and a semantic judgment task, these previous studies used different tasks in addition to the use of sentences with different familiarity levels that may have increased the cognitive demand and hence recruit different brain mechanism. In addition, whereas the present study focused on novel metaphoric expressions, these previous studies have focused mainly on conventional metaphoric sentences that are used in daily life, which are processed mainly by the LH. Their findings are therefore generally consistent with our results, indicating that processing familiar two-word expressions (both literal and metaphoric) is impaired when TMS is applied over the left IFG. However, it should be noted that Rapp, Leube, Erb, Grodd, and Kircher (2007) and Rapp et al. (2004), who apparently used novel metaphorical ("the lovers words are harp sounds") and literal ("the lovers words are lies") sentences, found stronger activation for the metaphorical sentences in left IFG and left temporal pole, but no significant differences in RH homologues.

For the conventional metaphoric expressions, there was a selective effect when rTMS was applied over the left IFG and a smaller effect when rTMS was applied over the right IFG. These regions were also involved in the efficient processing of literal word pairs. These findings are in line with previous results that have revealed the causal role of the left IFG in semantic processing (Nixon et al., 2004; Oliveri et al., 2004; Devlin et al., 2003). Our predictions for decreasing TMS effects in the left IFG for the meaningful expressions (i.e., greatest TMS effect for conventional metaphors, smaller for literal expressions and smallest for novel metaphors) were partially supported by the present results. Results of Experiment 2 showed similar TMS effects for conventional metaphors and literal expressions and no TMS effect for the novel metaphors at the left and right IFG. These findings emphasize the importance of distinguishing between more and less familiar linguistic expressions (i.e., literal and conventional metaphors vs. novel metaphors) or, more specifically, between familiar and unfamiliar metaphoric expressions. This dichotomy is supported by the career of metaphors hypothesis (Bowdle \& Gentner, 2005), which postulates that two different cognitive 
mechanism are involved in the processing of conventional as opposed to novel metaphors. Accordingly, conventional metaphors are understood preferentially via categorization processes whereas novel metaphors are understood via comparison processes, or as Bowdle and Gentner (2005, p. 199) state: "novel metaphors invite sense creation but conventional metaphors invite sense retrieval." Thus, it might be that the different cognitive processes (i.e., categorization vs. comparison) that mediate the comprehension of the familiar expressions (i.e., literal and conventional metaphoric expressions) and the novel metaphors, respectively, can explain the TMS effect over the left and right IFG for the familiar expressions but not for the novel metaphoric expressions. Note that, for the first time, we demonstrated here that anterior regions in the $\mathrm{LH}$ and $\mathrm{RH}$ are engaged in semantic processing that may only be limited to familiar semantic associations.

The most significant finding of the present study is the different pattern of responses to the novel metaphors by the two cerebral hemispheres. Thus, when two previously unfamiliar words that are conceptually capable of being linked are presented to an individual for the first time, the RH may be more likely to accept the possibility of such a linkage being legitimate than the LH. This finding is supported by a recent behavioral study that used the divided visual field technique for presenting novel metaphoric two-word expressions and unrelated word pairs (Faust \& Mashal, 2007). Although both types of stimuli were unfamiliar, $\mathrm{RH}$ sensitivity $\left(d^{\prime}\right)$ of the $\mathrm{RH}$ to the novel metaphoric expressions was significantly higher than that of the LH. Thus, the findings indicated higher ability in the RH to differentiate between unfamiliar but meaningful expressions from unfamiliar meaningless expressions. In accordance with previous rTMS studies that employed forced-choice tasks, in the present study, the effects of TMS were evident in the reaction time data, but did not affect accuracy, similar to that observed in previous rTMS studies that employed forced-choice tasks (c.f. Lavidor, Ellison, \& Walsh, 2003). The semantic system possesses a great deal of redundancy, making it unlikely that a focal TMS pulse would completely "knock out" a particular area.

As noted above, an interesting aspect of the present study's results is that the novel, unfamiliar, meaning combinations (i.e., UR condition) that were judged as unrelated in pretests did not show any RH TMS effect, although they could also be described as relying on the processing of novel, nonsalient semantic relations (see also Faust \& Mashal, 2007; Mashal et al., 2007 for similar findings). These findings seem to suggest that even novel metaphoric combinations often reflect some kind of systematic, although nonsalient, conceptual knowledge. According to Gibbs (1994), our basic conceptualizations of experience constrain how we think creatively and that novel metaphoric expressions taken from poetry, like those used in the present study, may have great intuitive appeal precisely because their understanding is based on "built in" conceptualization of our everyday experience. These claims suggest that the $\mathrm{RH}$ activates and integrates semantic relations in a highly flexible, yet selective, manner. Unfamiliar, unusual conceptual combinations are comprehended by this hemisphere only if they are, in a sense, appropriate and useful, and thus, reflect the involvement of truly creative linguistic mechanisms (e.g., Dietrich, 2004).

The TMS effects over the left IFG are consistent with predictions derived from the graded salience hypothesis (Giora, 2003). In particular, the LH appears to play a crucial role in interpreting salient meanings, those associated with the meaning of conventional metaphors and literal expressions. In contrast, the $\mathrm{RH}$ is critical for interpreting nonsalient meanings, those associated with novel metaphoric expressions and unrelated word pairs. The distinction between salient and nonsalient meaning was also obtained in an event-related potential study (Laurent, Denhires, Passerieux, Iakimova, \& Hardy-Bayl, 2006), in which the N400 amplitude was smaller for words that were related to the salient meaning of familiar idioms compared to words that were related to the nonsalient meaning of familiar idioms. Our findings are also consistent with the results of Schmidt et al. (2007).

A powerful prediction that emerges from the current study is that patients with damage to the $\mathrm{RH}$ in the right Wernicke's area are likely to show particular deficits in understanding novel combinations of word meanings. Although the processing of metaphors was tested in the past with right-brain-damaged patients (Chobor \& Schweiger, 1998; Rehak, Kaplan, \& Gardner, 1992; Brownell et al., 1990), the distinction we offer between conventional and novel metaphors was not applied.

In conclusion, the present findings indicate that novel metaphoric expressions taken from poetry might be processed differently from conventional metaphors and that the rPSTS plays a unique role in processing novel metaphors but not in the processing of conventional metaphoric expressions. In addition, stimulation of the left PSTS did not impair, and even facilitated, the processing of the nonsalient meanings. These findings may point to an important implication of $\mathrm{RH}$ propensity to activate and maintain a wide range of word meanings: the ability to integrate the meanings of several seemingly unrelated concepts into a new, meaningful linguistic expression. Because the comprehension of novel metaphors may require the activation of wider and less differentiated semantic fields, including nonsalient meanings, the findings support both the coarse semantic coding model (Jung-Beeman, 2005; Beeman, 1998) and the graded salience hypothesis (Giora 1997, 2003). We suggest that this type of hemispheric interaction be explicitly investigated in future studies. Such research could serve as an important example of how the LH language system is supported by the different kinds of semantic processes subserved by the $\mathrm{RH}$ and how the availability of both 
systems enables a wide variety of linguistic functions to be exploited as needed (Chiarello, 2003). We suggest that future research use the TMS technique to further explore the implications of the different roles of the two cerebral hemispheres in processing linguistic forms that are characterized by conventional as well as by unusual semantic relations.

\section{Acknowledgments}

This study was supported by the European Commission grant (RTN:LAB, MRTN-CT-2004-512141) awarded to G. P. and M. L. Additionally, N. M. and M. F. were supported by the Binational Science Foundation (BSF). We thank Vincent Walsh for enabling this venture.

Reprint requests should be sent to Michal Lavidor, Department of Psychology, the University of Hull, Hull HU6 7RX, UK, or via e-mail: M.Lavidor@hull.ac.uk.

\section{REFERENCES}

Ahrens, K., Liu, H. L., Lee, C. Y., Gong, S. P., Fang, S. Y., \& Hsu, Y. Y. (2007). Functional MRI of conventional and anomalous metaphors in Mandarin Chinese. Brain and Language, 100, 163-171.

Andoh, J., Artiges, E., Pallier, C., Riviere, D., Mangin, J. F., Cachia, A., et al. (2006). Modulation of language areas with functional MR image-guided magnetic stimulation. Neuroimage, 29, 619-627.

Balota, D. A., Cortese, M. J., Hutchison, K. A., Neely, J. H., Nelson, D., Simpson, G. B., et al. (2002). The English Lexicon Project: A web-based repository of descriptive and behavioral measures for 40,481 English words and nonwords. Retrieved from http://elexicon.wustl.edu/ on 5 October 2005.

Beck, B. (1987). Metaphor, cognition and artificial intelligence. In R. S. Haskell (Ed.), Cognition and symbolic structure: The psychology of metaphoric transformation (pp. 9-30). Norwood, NJ: Ablex.

Beeman, M. (1998). Coarse semantic coding and discourse comprehension. In M. Beeman \& C. Chiarello (Eds.), Right hemisphere language comprehension: Perspectives from cognitive neuroscience (pp. 255-284). Mahwah, NJ: Erlbaum.

Bottini, G., Corcoran, R., Sterzi, R., Paulesu, E. S. P., Scarpa, P., Frackoviak, R. S. J., et al. (1994). The role of the right hemisphere in the interpretation of the figurative aspects of language: A positron emission tomography activation study. Brain, 117, 1241-1253.

Bowdle, B. F., \& Gentner, D. (2005). The career of metaphor. Psychological Review, 112, 193-216.

Brownell, H. H., Simpson, T. L., Bihrle, A. M., Potter, H. H., \& Gardner, H. (1990). Appreciation of metaphoric alternative word meanings by left and right brain damaged patients. Neuropsychologia, 28, 375-383.

Burgess, C., \& Chiarello, C. (1996). Neurocognitive mechanisms underlying metaphor comprehension and other figurative language. Metaphor and Symbolic Activity, 11, 67-84.

Cacciary, C., \& Glucksberg, S. (1994). Understanding figurative language. In M. A. Gernsbacher (Ed.), Handbook of psycholinguistics (pp. 447-477). New York: Academic Press.

Chiarello, C. (1991). Interpretation of word meanings in the cerebral hemispheres: One is not enough. In P. J.
Schwanenflugel (Ed.), The psychology of word meanings (pp. 251-275). Hillsdale, NJ: Erlbaum.

Chiarello, C. (2003). Parallel systems for processing language: Hemispheric complementarity in the normal brain. In M. T. Banich \& M. Mack (Eds.), Mind, brain E language (pp. 229-247). Mahwah, NJ: Erlbaum.

Chobor, K., \& Schweiger, A. (1998). The comprehension of lexical ambiguity by brain-injured patients. In M. Paradis (Ed.), Pragmatics in neurolinguistic communication disorders. New York: Pergamon Press, Elsevier Science. Coltheart, M. (1981). The MRC psycholinguistic database. Quarterly Journal of Experimental Psychology, 33A, 497-505.

Devlin, J. T., Matthews, P. M., \& Rushworth, M. F. (2003). Semantic processing in the left inferior prefrontal cortex: A combined functional magnetic resonance imaging and transcranial magnetic stimulation study. Journal of Cognitive Neuroscience, 15, 71-84.

Dietrich, A. (2004). The cognitive neuroscience of creativity. Psychonomic Bulletin E Review, 11, 1011-1026.

Eviatar, Z., \& Just, M. A. (2006). Brain correlates of discourse processing: An fMRI investigation of irony and conventional metaphor comprehension. Neuropsychologia, 44, 2348-2359.

Faust, M., \& Lavidor, M. (2003). Convergent and divergent priming in the two cerebral hemispheres: Lexical decision and semantic judgment. Cognitive Brain Research, $17,585-597$.

Faust, M., \& Mashal, N. (2007). The role of the right cerebral hemisphere in processing novel metaphoric expressions taken from poetry: A divided visual field study. Neuropsychologia, 45, 860-870.

Floel, A., Buyx, A., Breitenstein, C., Lohmann, H., \& Knecht, S. (2005). Hemispheric lateralization of spatial attention in right- and left-hemispheric language dominance. Behavioural Brain Research, 158, 269-275.

Gazzaniga, M. S. (2000). Cerebral specialization and interhemispheric communication: Does the corpus callosum enable the human condition? Brain, 123, 1293-1326.

Gibbs, R. W. (1994). Figurative thought and figurative language. In M. A. Gernsbacher (Ed.), Handbook of psycholinguistics (pp. 411-446). New York: Academic Press.

Giora, R. (1997). Understanding figurative and literal language: The graded salience hypothesis. Cognitive Linguistics, 7, 183-206.

Giora, R. (2002). Literal vs. figurative language: Different or equal? Journal of Pragmatics, 34, 487-506.

Giora, R. (2003). On our mind: Salience, context and figurative language. New York: Oxford University Press.

Giora, R., \& Fein, O. (1999a). On understanding familiar and less familiar figurative language. Journal of Pragmatics, 31, 1601-1618.

Giora, R., \& Fein, O. (1999b). Irony: Context and salience. Metaphor and Symbol, 14, 241-257.

Giora, R., Zaidel, E., Soroker, N., Batori, G., \& Kasher, A. (2000). Differential effects of right- and left-hemisphere damage on understanding sarcasm and metaphor. Metaphor and Symbol, 15, 63-83.

Gough, P. M., Nobre, A. C., \& Devlin, J. T. (2005). Dissociating linguistic processes in the left inferior frontal cortex with transcranial magnetic stimulation. Journal of Neuroscience, 25, 8010-8016.

Jung-Beeman, M. (2005). Bilateral brain processes for comprehending natural language. Trends in Cognitive Sciences, 9, 512-518.

Jung-Beeman, M., Bowden, E. M., Haberman, J., Frymiare, J. L., Arambel-Liu, S., Greenblatt, R., et al. (2004). Neural activity when people solve verbal problems with insight. PLOS Biology, 2, 500-510. 
Laurent, J. P., Denhires, G., Passerieux, C., Iakimova, G., \& Hardy-Bayl, M. C. (2006). On understanding idiomatic language: The salience hypothesis assessed by ERPs. Brain Research, 1068, 151-160.

Lavidor, M., Ellison, A., \& Walsh, V. (2003). Examination of a split-processing model of visual word recognition: A magnetic stimulation study. Visual Cognition, 10, 341-362.

Loo, C. K., Taylor, J. L., Gandevia, S. C., McDarmont, B. N., Mitchell, P. B., \& Sachdev, P. S. (2000). Transcranial magnetic stimulation (TMS) in controlled treatment studies: Are some "sham" forms active? Biological Psychiatry, 47, 325-331.

Mashal, N., Faust, M., \& Hendler, T. (2005). The role of the right hemisphere in processing nonsalient metaphorical meanings: Application of principal components analysis to fMRI data. Neuropsychologia, 43, 2084-2100.

Mashal, N., Faust, M., Hendler, T., \& Jung-Beeman, M. (2007). An fMRI investigation of the neural correlates underlying the processing of novel metaphoric expressions. Brain and Language, 100, 115-126.

Nixon, P., Lazarova, J., Hodinott-Hill, I., Gough, P., \& Passingham, R. (2004). The inferior frontal gyrus and phonological processing: An investigation using rTMS. Journal of Cognitive Neuroscience, 16, 289-300.

Oldfield, D. J. (1971). The assessment of handedness: The Edinburgh Inventory. Neuropsychologia, 9, 97-113.

Oliveri, M., Romero, L., \& Papagno, C. (2004). Left but not right temporal involvement in opaque idiom comprehension: A repetitive transcranial magnetic stimulation study. Journal of Cognitive Neuroscience, 16, 848-855.

Oliveri, M., Turriziani, P., Carlesimo, G. A., Koch, G., Tomaiuolo, F., Panella, M., et al. (2001). Parieto-frontal interactions in visual-object and visual-spatial working memory: Evidence from transcranial magnetic stimulation. Cerebral Cortex, 11, 606-618.

Rapp, A. M., Leube, D. T., Erb, M., Grodd, W., \& Kircher, T. T. (2004). Neural correlates of metaphor processing. Cognitive Brain Research, 20, 395-402.

Rapp, A. M., Leube, D. T., Erb, M., Grodd, W., \& Kircher, T. T. (2007). Laterality in metaphor processing: Lack of evidence from functional magnetic resonance imaging for the right hemisphere theory. Brain and Language, 100, 142-149.

Rehak, A., Kaplan, J. A., \& Gardner, H. (1992). Sensitivity to conversational deviance in right-hemisphere-damaged patients. Brain and Language, 42, 203-217.

Rinaldi, M. C., Marangolo, P., \& Baldassari, F. (2004). Metaphor comprehension in right brain-damaged patients with visuoverbal and verbal material: A dissociation (re)considered. Cortex, 40, 479-490.

Schmidt, G. L., DeBuse, C. J., \& Seger, C. A. (2007). Right hemisphere metaphor processing? Characterizing the lateralization of semantic processes. Brain and Language, 100, 127-141.

Seyal, M., Ro, T., \& Rafal, R. (1995). Increased sensitivity to ipsilateral cutaneous stimuli following transcranial magnetic stimulation of the parietal lobe. Annals of Neurology, 38, 264-267.

Sotillo, M., Carretié, L., Hinojosa, J. A., Manuel, M., Mercado, F., López-Martín, S., et al. (2005). Neural activity associated with metaphor comprehension: Spatial analysis. Neuroscience Letters, 373, 5-9.

Stephan, K. E., Marshall, J. C., Friston, K. J., Rowe, J. B., Ritzl, A., Zilles, K., et al. (2003). Lateralized cognitive processes and lateralized task control in the human brain. Science, 301, 384-386.

Stewart, L., Meyer, B., Frith, U., \& Rothwell, J. (2001). Left posterior BA37 is involved in object recognition: A TMS study. Neuropsychologia, 39, 1-6.

Stringaris, A. K., Medford, N. C., Giampetro, V., Brammer, M. J., \& David, A. S. (2007). Deriving meaning: Distinct neural mechanisms for metaphoric, literal and non-meaningful sentences. Brain and Language, 100, 150-162.

Tompkins, C. A. (1990). Knowledge and strategies for processing lexical metaphor after right or left hemisphere brain damage. Journal of Speech and Hearing Research, 33, 307-316.

Vallesi, A., Shallice, T., \& Walsh, V. (2007). Role of the prefrontal cortex in the foreperiod effect: TMS evidence for dual mechanisms in temporal preparation. Cerebral Cortex, 17, 466-474. 